\title{
Application of Commuting Mapping in b-metric space
}

\author{
Neelam Yadav ${ }^{1}$, Piyush Kumar Tripathi ${ }^{2}$, Alok Kumar Agrawal ${ }^{3}$ \\ Deptt. of Mathematics, Amity School of Applied Sciences, Amity University, (U.P), India ${ }^{1,2,3}$, \\ Research Scholar ${ }^{1}$,Associate Professor ${ }^{2,3}$ \\ Email:neelam3376@yahoo.co.in. ${ }^{1}$, pktripathi@lko.amity.edu ${ }^{2}$, akagrawal@lko.amity.edu ${ }^{3}$
}

\begin{abstract}
Theory of contraction principle is based on mathematical systems and these are over idealistic which have prompted the scientists to evolve new techniques and mathematical apparatus to understand real life phenomena and interpret them correctly within the frame work of needed objectives. In this paper, two theorems have been proved using the commutative property of mappings in b-metric space.
\end{abstract}

Index Terms-Contraction principle; b-metric space, commuting mapping, fixed point, coincidence point.

\section{INTRODUCTION}

Fixed point theory is important field in nonlinear analysis, also in digital image processing as well as in the field of digital signal processing. Banach introduced Banach contraction principle [1] which guarantees the existence and uniqueness of fixed point of contraction mapping in complete metric space. This has been generalized and extended by several researchers [2-5] in different spaces. Following the Banach contraction principle, Nadler [2] introduced the concept of set valued contractions and established that these possess a fixed point in complete metric space. The concept of b-metric space was introduced by Stefan Czerwik [3] as a generalization of metric space. Kir and Kiziltunc [6] studied Kannan and Chatterjea type contractive mappings and showed that these have unique fixed point in b-metric space. Ran and Reurings proved some results on the existence of fixed points in partially ordered sets and are applied to the resolution of matrix equations [8]. Jacek [9] generalized the Banach contraction principle to mappings on a metric space endowed with a graph and applied a theoremon the convergence of successive approximations for some linear operators on a Banach space. Pfeffer [10] gave a proposition depicting an interdependence between the commuting mapping and the fixed point concepts, proved that any involution $r$ of a circle $S$ has a fixed point iff there exists a free involution of $\mathrm{S}$ which commutes with $r$. Jungck [11] introduced the concept of contraction mapping for two functions and proved coincidence and common fixed point theorems for such mappings. Jungck [12] highlighted interdependence concept of [10] in a general context.

\section{PRELMINARIES}

Definition 1[1]: In the metric space $(X, d)$, a mapping $T: X \rightarrow X$ is said to be contraction if $\exists k \in[0,1)$, such that $\forall x, y \in X ; d(T x, T y) \leq k d(x, y)$

Definition 2: Let $X$ be a non-empty set and let $d: X \times X \rightarrow \mathbb{R}_{+}$, then, $(X, d)$ is b-metric space if the following conditions hold:

(1) $d(x, y)=0 \Leftrightarrow x=y$;

(2) $d(x, y)=d(y, x)$;

(3) $d(x, z) \leq k\{d(x, y)+d(y, z)\} \forall x, y, z \in X, k \geq 1$ If $k=1$, every b-metric space is metric space.

Proposition [10]: Let $f$ be a mapping of a set $X$ into itself. Then $f$ has a fixed point iff there is a constant map $h: X \rightarrow X$ which commutes with $f$ (i.e., $h(f(x))=f(h(x))$ for all $x$ in $X)$.

Theorem [11]: Consider a complete metric space $(X, d), f$ be a continuous mapping into itself. Then $f$ has a fixed point in $X$ iff $\exists \gamma \in(0,1)$ and a mapping $g: X \rightarrow X$ which commutes with $f$ and satisfies $(f(g(X) \subset f(X)$ and $d(g(x), g(y)) \leq$

$$
\gamma d(f(x), f(y)) \forall x, y \in X
$$

then $f$ and $g$ have a unique common fixed point.

\section{MAIN RESULTS}

Theorem 3.1. Let $(X, d)$ be a metric space and $Y$ be any set. Suppose $f, g: Y \rightarrow X$ are mapping, such that (1) $f(Y) \subseteq g(Y)$

(2) $d(f x, f y) \leq$ $a \max \{d(g x, f x), d(g y, f y), d(g x, g y)\}+$
$b\{d(g x, f y)+d(g y, f x)\}$

(3) Either $g(Y)$ or $f(Y)$ is complete, then $f$ and $g$ have coincidence point.

Proof: Let $x_{0} \in X$ and $\left\{g x_{n}\right\}_{n=1}^{\infty}$ be a sequence in $\mathrm{X}$ defined by the recursion $g x_{n}=f x_{n-1}=f^{n} x_{0} \quad \mathrm{n}=1,2,3,4 \ldots \ldots$ 


\section{Available online at www.ijrat.org}

$$
\begin{gathered}
d\left(g x_{n}, g x_{n+1}\right)=d\left(f x_{n-1}, f x_{n}\right) \\
\leq a \max \left\{d\left(g x_{n-1}, f x_{n-1}\right),\right. \\
\left.d\left(g x_{n}, f x_{n}\right), d\left(g x_{n-1}, g x_{n}\right)\right\} \\
+b\left\{d\left(g x_{n-1}, f x_{n}\right)+d\left(g x_{n}, f x_{n-1}\right)\right\} \\
d\left(f x_{n-1}, f x_{n}\right) \leq a \max _{2}\left\{d\left(g x_{n-1}, g x_{n}\right),\right. \\
\left.\quad d\left(g x_{n}, g x_{n+1}\right), d\left(g x_{n-1}, g x_{n}\right)\right\} \\
+b\left\{d\left(g x_{n-1}, g x_{n+1}\right)+d\left(g x_{n}, g x_{n}\right)\right\} \\
\leq a \max \left\{d\left(g x_{n-1}, g x_{n}\right),\right. \\
\left.d\left(g x_{n}, g x_{n+1}\right)\right\} \\
+b\left\{d\left(g x_{n-1}, g x_{n+1}\right)\right\}
\end{gathered}
$$

$d\left(g x_{n}, g x_{n+1}\right)$

$\leq a \max \left\{d\left(g x_{n-1}, g x_{n}\right), d\left(g x_{n}, g x_{n+1}\right)\right\}+$ $s b\left\{d\left(g x_{n-1}, g x_{n}\right)+d\left(g x_{n-1}, g x_{n+1}\right)\right\}$

$\leq a M_{1}+s b\left\{d\left(g x_{n-1}, g x_{n}\right)+d\left(g x_{n-1}, g x_{n+1}\right)\right.$

where $M_{1}=\max \left\{d\left(g x_{n-1}, g x_{n}\right), d\left(g x_{n}, g x_{n+1}\right)\right\}$

Case I: If $M_{1}=d\left(g x_{n}, g x_{n+1}\right)$, then

$$
\begin{aligned}
& d\left(g x_{n}, g x_{n+1}\right) \leq a d\left(g x_{n}, g x_{n+1}\right) \\
&+s b\left\{d\left(g x_{n-1}, g x_{n}\right)\right. \\
&\left.+d\left(g x_{n-1}, g x_{n+1}\right)\right\} \\
&(1-a-s b) d(\left.g x_{n}, g x_{n+1}\right) \leq s b\left\{d\left(g x_{n-1}, g x_{n}\right)\right\} \\
& d\left(g x_{n}, g x_{n+1}\right) \leq \frac{\mathrm{sb}}{(1-a-s b)}\left\{d\left(g x_{n-1}, g x_{n}\right)\right\} \\
& \leq \mathrm{k}\left\{d\left(g x_{n-1}, g x_{n}\right)\right\}, \quad k<1
\end{aligned}
$$

repeatedly using the above result

$$
\leq k^{3}\left\{d\left(g x_{n-3}, g x_{n-2}\right)\right\} \ldots \ldots \ldots
$$

$$
\leq k^{n}\left\{d\left(g x_{0}, g x_{1}\right)\right\} \quad k<1
$$

Case II: If $M_{1}=d\left(g x_{n-1}, g x_{n}\right)$, then

$d\left(g x_{n}, g x_{n+1}\right) \leq a d\left(g x_{n-1}, g x_{n}\right)$ $+s b\left\{d\left(g x_{n-1}, g x_{n}\right)\right\}$

$(1-s b) d\left(g x_{n}, g x_{n+1}\right)$ $+s b\left\{d\left(g x_{n-1}, g x_{n+1}\right)\right\}$

$$
\begin{aligned}
& \leq a d\left(g x_{n-1}, g x_{n}\right) \\
& +s b\left\{d\left(g x_{n-1}, g x_{n}\right)\right\}
\end{aligned}
$$

$(1-s b) d\left(g x_{n}, g x_{n+1}\right) \leq(a+s b)\left\{d\left(g x_{n-1}, g x_{n}\right)\right\}$

$$
d\left(g x_{n}, g x_{n+1}\right) \leq \frac{\mathrm{a}+\mathrm{sb}}{(1-s b)}\left\{d\left(g x_{n-1}, g x_{n}\right)\right\}
$$$$
\leq \mathrm{k}\left\{d\left(g x_{n-1}, g x_{n}\right)\right\}, k<1
$$

repeatedly using the above result

$$
\leq k^{2}\left\{d\left(g x_{n-2}, g x_{n-1}\right)\right\}
$$

$$
\leq k^{n}\left\{d\left(g x_{0}, g x_{1}\right)\right\} \quad k<1
$$

Thus $f$ is a contractive mapping. Now we show that $\left\{g x_{n}\right\}_{n=1}^{\infty}$ is a Cauchy sequence in $X$.

Let $m, n \in \mathbb{N}, \quad m>n$

$$
\begin{gathered}
d\left(g x_{n}, g x_{m}\right) \leq s\left\{d\left(g x_{n}, g x_{n+1}\right)\right\} \\
\left.+d\left(g x_{n+1}, g x_{m}\right)\right\} \\
\leq s\left\{d\left(g x_{n}, g x_{n+1}\right)\right\}+s\left\{s \left[d\left(g x_{n+1}, g x_{n+2}\right)\right.\right. \\
\left.\left.+d\left(g x_{n+2}, g x_{m}\right)\right]\right\} \\
\leq s\left\{d\left(g x_{n}, g x_{n+1}\right)\right\}+s^{2}\left\{d\left(g x_{n+1}, g x_{n+2}\right)\right\} \\
+s^{2}\left\{d\left(g x_{n+2}, g x_{m}\right)\right\} \\
\leq s\left\{d\left(g x_{n}, g x_{n+1}\right)\right\}+s^{2}\left\{d\left(g x_{n+1}, g x_{n+2}\right)\right\} \\
+s^{3}\left\{d\left(g x_{n+2}, g x_{n+3}\right)\right\} \ldots \ldots . .
\end{gathered}
$$

$$
\begin{gathered}
\quad \leq s k^{n}\left\{d\left(g x_{0}, g x_{1}\right)\right\}+s^{2} k^{n+1}\left\{d\left(g x_{0}, g x_{1}\right)\right\} \\
+s^{3} k^{n+2}\left\{d\left(g x_{n+2}, g x_{m}\right)\right\} \ldots \ldots
\end{gathered}
$$

since $k<1$,

$\lim n \rightarrow \infty \frac{s k^{n}}{1-s k} d\left(g x_{0}, g x_{1}\right)=0$ as $n, m$

Hence $\left\{g x_{n}\right\}_{n=1}^{\infty}$ is a Cauchy sequence in $X$.

Suppose $g(Y)$ is complete. Since $\left\{g x_{n}\right\}_{n=1}^{\infty}$ is a

Cauchy sequence, $\left\{g x_{n}\right\}$ converges to $p \in g(Y)$, i.e

$\exists z \in Y$, such that $g z=p$

Put $x=x_{n}, y=z$ in condition (2) of the theorem, we get:

$d\left(f x_{n}, f z\right)$

$\leq a \max \left\{d\left(g x_{n}, g z\right), d(g z, f z), d\left(g x_{n}, g z\right)\right\}$

$+b\left\{d\left(g x_{n}, f z\right)+d\left(g z, f x_{n}\right)\right\}$

for $n \rightarrow \infty, f x_{n} \rightarrow p$,

$d(p, f z) \leq a \max \{d(p, p), d(p, f z), d(p, p)\}$

$$
+b\{d(p, f z)+d(p, p)\}
$$

$d(p, f z) \leq a\{d(p, f z)\}+b\{d(p, f z)\}$

$(1-a-b)\{d(p, f z)\}$

$\leq 0$

$\therefore\{d(p, f z)\}=0$

$$
(1-a-b) \neq 0 ;\{d(p, f z)\}>0
$$

$\Rightarrow f z=p$

i.e. $\mathrm{z}$ is the coincidence point of $f$ and $g$.

Now, if $f(Y)$ is complete, then $f x_{n} \rightarrow p \in f(Y) \subseteq$ $g(Y)$

Hence, as above $\mathrm{z}$ is coincidence point of $f$ and $g$.

Theorem 3.2. Let $(X, d)$ be a b-metric space.

Suppose $f, g: X \rightarrow X$ are mappings, such that

(1) $f(X) \subseteq g(X)$

(2) $d(f x, f y) \leq$

$$
\begin{gathered}
a \max \{d(g x, f x), d(g y, f y), d(g x, g y)\}+ \\
b\{d(g x, f y)+d(g y, f x)\}
\end{gathered}
$$

(3) Either $g(X)$ or $f(X)$ is complete.

(4) $f$ and $g$ are commuting at their coincidence point then $f$ and $g$ have unique fixed point.

Proof: In the previous theorem, if we take $Y=X$, then we get sequence $g x_{n}=f x_{n-1}$, is a Cauchy sequence. In the case of $g(X)$ or $f(X)$ is complete, as in previous theorem, we get $f z=g z=p$

Since, $f$ and $g$ are commuting at their point of coincidence, so $f g z=g f z \Rightarrow f p=g p$

Put $x=z, y=f(z)$ in condition (2) of theorem, $d(f z, f f z)$

$\leq a \max \{d(g z, f z), d(g f z, f f z), d(g z, g f z)\}$

$+b\{d(g z, f f z)+d(g f z, f z)\}$

$d(p, f p) \leq a \max \{d(p, p), d(g p, f p), d(p, g p)\}$ $+b\{d(p, f p)+d(g p, p)\}$ 


\section{Available online at $w w w . i j r a t . o r g$}

$$
\begin{aligned}
& \leq a\{d(p, g p)\}+b\{d(p, f p) \\
& \quad+d(g p, p)\} \\
& \leq a\{d(p, g p)\}+\frac{b}{s}\{d(f p, g p)\}
\end{aligned}
$$

(using triangle inequality)

$$
d(p, f p) \leq a\{d(p, g p)\}
$$

$(1-a)\{d(p, f p)\} \leq 0,(1-a) \neq 0$

$d(p, f p)=0 \quad \Rightarrow p=f p=g p$

$p$ is the common fixed point of $f$ and $g$

For uniqueness:

Suppose $p$ and $q$ are two common fixed points of

$f$ and $g$, then $f p=g p=p$

also, $f q=g q=q$

Put $x=p, y=q$ in condition (2) of the theorem,

$$
d(f p, f q)
$$$$
\leq a \max \{d(g p, f p), d(g q, f q), d(g p, g q)\}
$$$$
+b\{d(g p, f q)+d(g q, f p)\}
$$

$d(p, q) \leq a d(p, q)+2 b\{d(p, q)\}$

$$
\begin{gathered}
(1-a-2 b) d(p, q) \leq 0, \quad(1-a-2 b) \neq 0 \\
d(p, q)=0 \Rightarrow p=q
\end{gathered}
$$

Corollary: Let $(X, d)$ be a complete b-metric space.

Let $f: X \rightarrow X$ be a mapping, such that

$$
\begin{gathered}
d(f x, f y) \leq a \max \{d(x, f x), d(y, f y), d(x, y)\} \\
+b\{d(x, f y)+d(y, f x)\}
\end{gathered}
$$

where $p, q \geq 0, \quad a+2 s b \leq 1 \forall x, y \in X, \quad s \geq 1$ then, $f$ has a unique fixed point.

Proof: In theorem 2, if we take

$g=I$ (Identity mapping) then result of [7] follows.

\section{REFERENCES}

[1] Banach. S. (1922): Sur les operations dans les ensembles abstraits et leur application aux equations integrals. Fundam. Math., 3, pp. 133181. (in French).

[2] Nadler S. B. (1969): Multivalued Contraction Mappings. Pacific J. Math., 30, pp. 475-488.

[3] Czerwik, S. (1993): Contraction mappings in bmetric spaces. Acta Math. Inform. Univ.Ostraviensis, 1, pp. 5-11.

[4] Babu, G.V.R., Sailaja, P.D. (2011): A Fixed Point Theorem of Generalized Weakly Contractive Maps in Orbitally Complete Metric Spaces. Thai Journal of Mathematics, 9 (1), pp. 1-10.

[5] Hussain, N., Kutbi, M.A., Salimi, P. (2014): Fixed point theory in $\alpha$-complete metric spaces with applications. Abstr. Appl. Anal., 11 pages.

[6] Mehmet K. and Kiziltunc H. (2013): On Some Well Known Fixed Point Theorems in b-metric spaces. Turkish Journal of Analysis and Number Theory, 1(1), pp. 13-16.

[7] Agrawal S., Qureshi K., Verma J. (2016): A Fixed Point Theorem for b-metric space", International Journal of Pure and Applied Mathematical Science, 9 (1), pp. 45-50.
[8] Ran A.C.M. and Reurings M.C.B. (2004): A fixed point theorem in partially ordered sets and some applications to matrix equations. Proc. Amer. Math. Soc. 132, pp. 1435-1443.

[9] Jacek J. (2008): The Contraction Principle for Mappings on a Metric Space with a Graph. Proceedings of the American Mathematical Society, 136 (4), pp. 1359-1373.

[10] Pfeffer W. F. (1974): More on involutions of a circle. The American Mathematical Monthly, 81, pp. 613-616.

[11]Jungck G. (1966): Commuting mappings and common fixed points. The American Mathematical Monthly, 73, pp. 735-738.

[12] Jungck G. (1976): Commuting Mappings and Fixed Points. The American Mathematical Monthly, 83 (4), pp. 261-263. 